\title{
Infection after implantation of pulsatile mechanical circulatory support devices
}

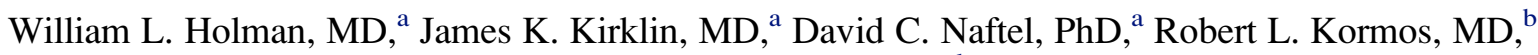 \\ Patricia Desvign-Nickens, $\mathrm{PhD},{ }^{\mathrm{c}}$ Margarita T. Camacho, $\mathrm{MD},{ }^{\mathrm{d}}$ and Deborah D. Ascheim, $\mathrm{MD}^{\mathrm{e}}$
}

\begin{abstract}
Objective: INTERMACS is a registry of mechanical circulatory support devices sponsored by the National Institutes of Health. This analysis uses INTERMACS data to define the time course, incidence, and outcome of infection adverse events focusing on the first 3 months after implant.
\end{abstract}

\begin{abstract}
Methods: Patients entered into INTERMACS from June 23, 2006, to September 30, 2008, were analyzed. Preimplant data (demographics, hemodynamics, and laboratory values), infection adverse events, and other outcomes were recorded. Infection adverse events were analyzed to compare infection rates in subgroups of patients and define risk factors for death.
\end{abstract}

\begin{abstract}
Results: The analysis was confined to pulsatile mechanical circulatory support devices. A total of 593 patients from 88 institutions were entered. Infection was a relatively common event within the first 3 months of implant and was significantly $(P=.005)$ more common in patients with biventricular assist devices than in patients with left ventricular assist devices, although the prevalence of infection equalized in months 4 to 12 . Infection had a significant adverse effect on survival. Independent risk factors for death included support with a biventricular assist device, older age, severity of patient illness implantation of the device (INTERMACS level 1), and higher blood urea nitrogen.
\end{abstract}

Conclusions: Infection remains a relatively frequent adverse event and is associated with decreased survival. Interventions to prevent infection that focus on the preoperative and immediate postoperative periods are the ones most likely to achieve success by diminishing the incidence of infection during the initial 3 months after implantation. Rotary (continuous-flow) pumps are expected to have lower infection rates, but this remains to be seen. (J Thorac Cardiovasc Surg 2010;139:1632-6)

f Supplemental material is available online.

The Interagency Registry for Mechanically Assisted Circulatory Support (INTERMACS) is a national registry for patients who receive a durable mechanical circulatory support device (MCSD) for advanced medically refractory heart failure. ${ }^{1}$ Only MCSDs approved by the United States Food and Drug Administration (FDA) are included in this registry. A list of the MCSDs represented in this analysis is presented in Appendix E1.

Infection, mechanical failure, and thromboembolic complications were recognized early in the development of MCSDs to be likely sources of morbidity and mortality. ${ }^{2}$

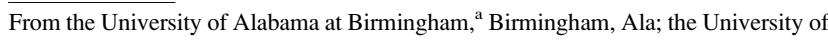
Pittsburgh, ${ }^{\mathrm{b}}$ Pittsburgh, Pa; the National Heart, Lung, and Blood Institute, ${ }^{\mathrm{c}}$ Rockville, Md; the Newark Beth Israel Medical Center, ${ }^{\mathrm{d}}$ Newark, NJ; and the Mount Sinai School of Medicine, ${ }^{\mathrm{e}}$ New York, NY.

Disclosures: Margarita T. Camacho reports fees and grant support from Thoratec.

Received for publication Sept 29, 2009; revisions received Dec 16, 2009; accepted for publication Jan 2, 2010; available ahead of print April 5, 2010.

Address for reprints: William L. Holman, MD, Room 719 ZRB, 703 19th St S,

Birmingham, AL 35294-0007 (E-mail: wholman@uab.edu).

$0022-5223 / \$ 36.00$

Copyright (c) 2010 by The American Association for Thoracic Surgery doi:10.1016/j.jtcvs.2010.01.014
This has proven to be the case, as shown in individual center experiences and multicenter studies, ${ }^{3-9}$ although advances in engineering, patient selection, and patient management are improving patient outcomes. ${ }^{10-13}$

Before initiating data collection for INTERMACS, a collaborative group of physicians, nurses, members of the regulatory community, including the FDA, and representatives from industry defined a list of adverse events (AEs) for this prospectively gathered database. Definitions for the AEs are available from the INTERMACS Web site (www.intermacs. org). The present study focuses on an analysis of infection AEs as listed in Appendix E2 during a defined period of time when essentially all implants of FDA-approved pumps were pulsatile devices. The purpose of the analysis was to define the time course, incidence, outcome, and risk factors for infection in MCSD recipients. The information will serve as a basis for the development of prophylactic and therapeutic measures aimed at diminishing the incidence and impact of infection in patients with MCSDs. Moreover, it will be possible to compare the rates and consequences of infection for pulsatile and continuous-flow (rotary) pumps as continuous-flow devices receive FDA approval.

\section{METHODS}

Prospectively gathered data were collected from MCSD implants that took place from June 23, 2006, to September 30, 2008 ( $\mathrm{n}=593$ patients). 


\section{Abbreviations and Acronyms \\ $\mathrm{AE} \quad=$ adverse event \\ BiVAD = biventricular assist device \\ FDA $\quad=$ Food and Drug Administration \\ INTERMACS $=$ Interagency Registry for \\ Mechanically Assisted \\ Circulatory Support
MCSD $=$ mechanical circulatory support device \\ VAD $\quad=$ ventricular assist device}

The numbers and location (left ventricular assist device [VAD] vs biventricular assist device [BiVAD]) for devices implanted during this time are listed in Table E1 along with the prevalence of infection AEs. Total artificial heart implants, isolated rotary VADs (ie, MicroMed DeBakey Child left VAD; MicroMed Technology, Inc, Houston, Tex), and isolated right VAD implants were uncommon relative to pulsatile BiVAD and left VAD implants. Isolated right $\mathrm{VAD}$, rotary $\mathrm{VAD}$, and total artificial heart implants were therefore not included in the present analysis of infection AEs. The indication for VAD use was determined before implant and was classified as bridge to transplantation, destination therapy (ie, a permanent implant in a patient not considered to be a candidate for transplantation), or bridge to recovery of the native heart.

During the period of this analysis, there were 88 institutions entering data. Participating centers are listed on the INTERMACS Web site. This list changes over time as centers are removed and added to the actively enrolling institutions.

Data were transmitted from sites using a Web-based system to a secure server administered by the United Network for Organ Sharing. The study sites and the central data processing and analyzing institutions received institutional review board approval before initiating the collection of data. The data coordinating center at University of Alabama at Birmingham provided data analysis. The variables specific to infection included the microbial etiology (bacterial, fungal, viral, protozoan, or unknown), the site of infection (if known), a determination of whether the infection was patient-related or device-related, and the therapy used for infection (drug only, drug and surgical, surgical only, or unknown).

The specific data gathered to describe patient demographics, hemodynamics, laboratory values, and other outcome events can be found at the INTERMACS Web site. The INTERMACS data were checked for completeness by the central collection facility (the United Network for Organ Sharing). Values that fell outside predetermined limits were validated with their site of origin. $\mathrm{AE}$ reporting forms are reviewed by two physicians from the INTERMACS community. Differences of opinion between the reviewers were adjudicated by members of the INTERMACS AE committee before final decisions were made on the classification of individual AEs. Source documents were not routinely available for verification or adjudication.

Actuarial depictions of data were used to compare subgroups for infection rates and survival. It was recognized that repeated infections can occur in individual patients. Therefore, the time to first infection was analyzed along with cumulative infection AEs within the population of MCSD recipients. A multivariable analysis was performed to define preimplant risk factors that were independent predictors for cumulative infections. The variables included in the analysis were patient demographics, hemodynamics, patient medical history, INTERMACS patient profile (severity of illness before implant), device strategy (left VAD versus BiVAD), and blood chemistry data. Hazard ratios were defined in addition to $P$ values for these independent risk factors.

\section{RESULTS}

A total of 593 patients were prospectively entered into the INTERMACS database between June 23, 2006, and September 30, 2008. These patients came from 88 institutions and were predominantly white men with an average age at implant of 51.5 years and a range of 19.8 to 79.0 years (Table E2). The prevalence of infection was similar for left VADs and BiVADs and was in line with the prevalence of infection previously reported for single institution and multicenter trials. ${ }^{14}$ The cumulative infection rates were similar between men and women, but the infection rate was significantly influenced by age (Figure 1). The majority $(87 \%)$ of infections were bacterial in origin with smaller percentages of fungal $(9 \%)$, viral $(1 \%)$, protozoal $(0.3 \%)$, or unknown $(2 \%)$ infections.

The most common preimplant INTERMACS level was level 1 (critical cardiogenic shock) and was reported for $42 \%$ of implanted patients. Lesser degrees of patient illness and acuity were seen in the remaining patients, although $38 \%$ were listed as INTERMACS level 2 (ie, progressive decline). The INTERMACS classification had a significant adverse affect on cumulative infection episodes across the initial 18 months after implant (Figure 2). This difference was most prominent during the first 3 months of implant, when level 1 patients had nearly 1.5 infection episodes per patient whereas levels 2 through 7 had less than 1.0 infection episodes per patient. An increase in infections episodes was seen between months 18 and 24 for INTERMACS levels 2 and levels 4 through 7 (Figure 2).

Infection episodes were most commonly related to positive blood cultures ( $32 \%$ of all infections) or driveline infections (21\% of all infections) (Table E3). Of note, $15 \%$ of reported infections were from other sources identified in text fields and were not included in these analyses. Devicerelated infections (driveline, pump pocket, and pump interior) occurred in 194 of 682 (28.4\%) of infection episodes.

The cumulative infection rate per patient was significantly higher in patients who received BiVAD support as compared with isolated left VAD support (Figure 3). Infection episodes in patients with BiVAD support were more common in the first 3 months of support as compared with the 4- to 6-month, 7- to 9-month, and 10- to 12-month intervals after implant (Figure E1). By the 10- to 12-month time frame, the rate of infection for left VADs was increasing.

Infection had a significant adverse affect on survival, and patients with an infection AE during the first month after implant had significantly worse survival than patients who did not have their first infection $\mathrm{AE}$ until the second or third month after implant (Figure 4). Moreover, patients requiring BiVAD support who had an infection within the first 3 months after implant had significantly worse survival than patients who had isolated left VAD support and an infection $\mathrm{AE}$ within the first 3 months of support (Figure 5). 
Cumulative Infection Events by Age (LVAD Only)

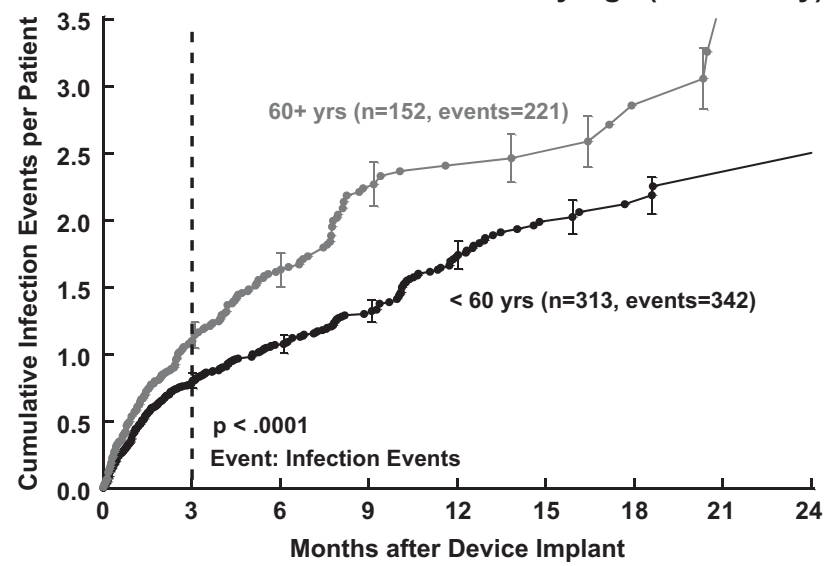

FIGURE 1. Cumulative infection events by age (left ventricular assist device only).

The multivariable analysis of preimplant risk factors for cumulative postimplant infections showed that use of BiVADs, older age, INTERMACS level 1 (critical cardiogenic shock), and higher blood urea nitrogen values were all predictors of a higher cumulative number of infection AEs (Table E4). Use of BiVADs, INTERMACS level 1, and higher blood urea nitrogen values can be viewed as indicators of more advanced heart failure or acute decompensation of chronic heart failure. The hypothesis of whether conversion to a higher INTERMACS level before implant via intensive preimplant medical management is intriguing, but it could not be evaluated from the data collected for this analysis.

\section{DISCUSSION}

Device reliability, thromboembolic complications, and infection were identified during the early phase of MCSD de-

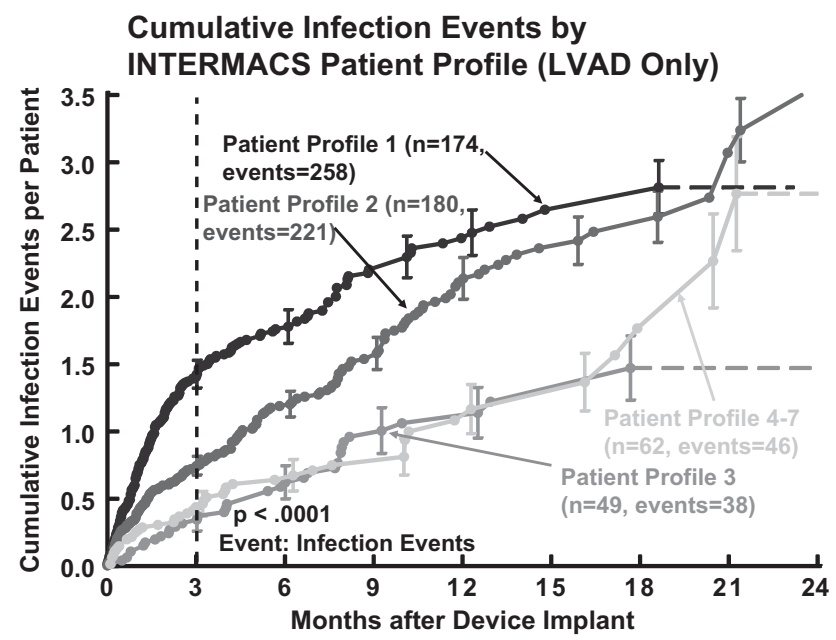

FIGURE 2. Cumulative infection events by INTERMACS patient profile (left ventricular assist device only).

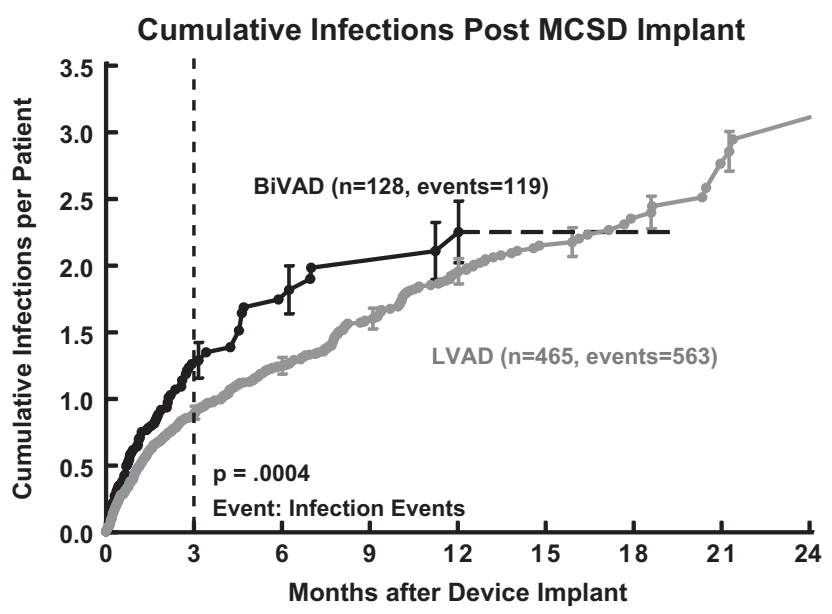

FIGURE 3. Cumulative infections after mechanical circulatory support device implant. BiVAD, Biventricular assist device; $L V A D$, left ventricular assist device.

velopment as the most likely obstacles to the successful use of MCSDs in prolonging survival and improving the quality of life for patients with advanced heart failure. ${ }^{2}$ These predictions proved correct, although continued improvements in device design, patient selection, and patient management promise to diminish their incidence and importance. ${ }^{10-13}$

During the era of this study, nearly all of the devices implanted were pulsatile pumps rather than continuous-flow (rotary) pumps. Pulsatile MCSDs are larger than rotary MCSDs. In addition, the implanted pulsatile pumps in this analysis have larger diameter and more rigid drivelines than rotary pumps to accommodate a channel that vents air displaced by the pumping sac. These considerations may influence the incidence and severity of device-related infections (eg, pump pocket and driveline infections). On the basis of experiences from single center and individual device

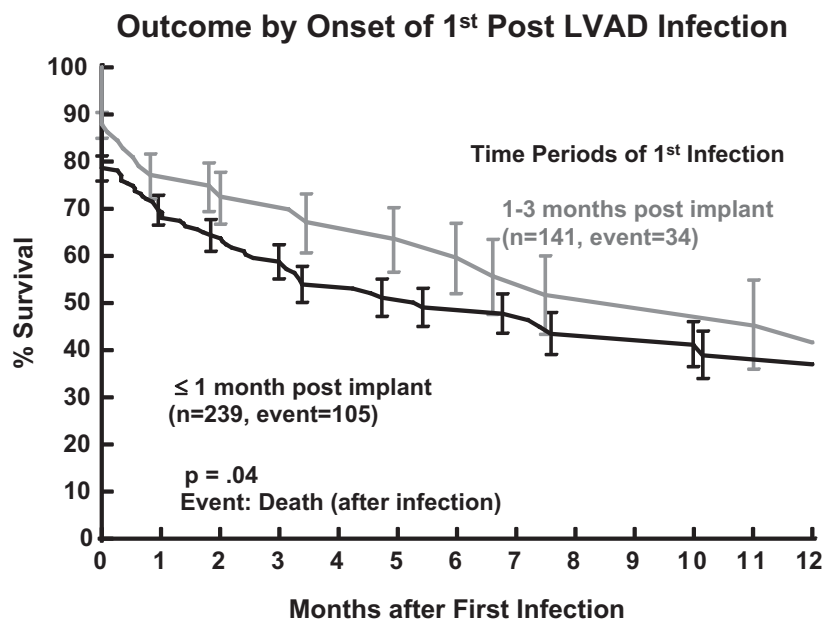

FIGURE 4. Outcome by onset of first infection after implantation of left ventricular assist device. 


\section{Outcome after Early Infection post MCSD Implant}

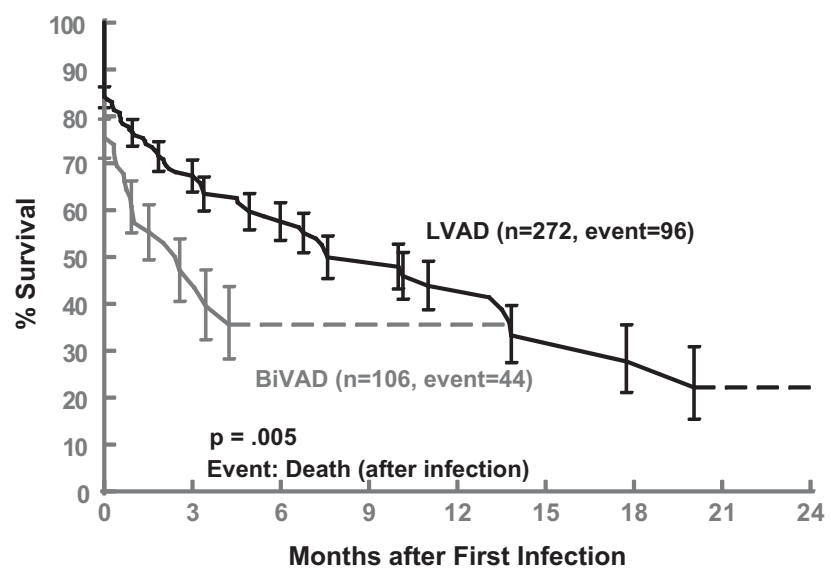

FIGURE 5. Outcome after early infection after implantation of mechanical circulatory support device. BiVAD, Biventricular assist device; $L V A D$, left ventricular assist device.

trials published to date for rotary pumps, INTERMACS may document a lower incidence of infection AEs for rotary pumps as their results enter the INTERMACS Registry.

The Randomized Evaluation of Mechanical Assistance for the Treatment of Congestive Heart Failure (REMATCH) Trial showed that an implanted pulsatile left VAD (HeartMate I; Thoratec Corporation, Pleasanton, Calif) provided superior survival ( $48 \%$ reduction in risk of death) as compared with optimal medical management for patients with advanced heart failure. However, infection AEs were relatively common in this group of patients (0.60 events/ patient-year for sepsis; 0.39 events/patient-year for local infection). There was a $28 \%$ (95\% confidence interval $15 \%-$ $38 \%$ ) probability of left VAD infection within 3 months after implant, and sepsis was the most common mode of death in patients who were randomized to MCSD therapy (17/41 patients). ${ }^{15}$ Further examination of the REMATCH Trial in a post hoc analysis of infection complications showed that the instantaneous risk (ie, hazard) for sepsis was highest within the first 30 days after device implant. ${ }^{16}$ No late rising phase of risk was identified in this study, although many of the patients required device replacement between 18 and 24 months after implant. The post hoc analysis showed an adverse effect of sepsis on survival with $60 \%$ 1-year and $38 \%$ 2-year survival for left VAD patients without sepsis versus a 39\% 1-year and 8\% 2-year survival for left VAD patients with an episode of sepsis $(P=.06)$. Interestingly, a subgroup of hospitals in this multicenter trial experienced fewer episodes of sepsis than other hospitals independent of other risk factors $(P=.012 ; 95 \%$ confidence interval $1.3-$ 7.0 ), suggesting that local surgical and nursing practice may have important effects on infection rate and survival for patients with implanted pulsatile left VADs for the indication of destination therapy.
The present analysis of the INTERMACS database found that infection AEs were most common within the first 3 months after implant and that the presence of infection had a statistically significant negative influence on survival. Furthermore, it appears that certain subgroups of patients (eg, patients with critical cardiogenic shock) are at highest risk for infection AEs. This is similar to prior analyses of the INTERMACS database that showed that indicators of right heart failure (eg, requirement for BiVAD support, hepatic dysfunction characterized by elevated bilirubin or ascites) and acute circulatory decompensation (eg, INTERMACS level 1) were associated with death. ${ }^{17}$ However, it also appears that after the initial 3 months of implant the difference in cumulative infections diminishes for BiVAD patients as compared with the left VAD recipients, perhaps because those patients with preoperative biventricular failure achieve a compensated state on BiVAD support.

Driveline infections were a relatively common event $(21 \%$ of infection AEs). It is possible that eliminating the need for a percutaneous driveline will substantially diminish the number of infection AEs in MCSD recipients. Obstacles to the elimination of the driveline include the engineering complexity and reliability demands for fully implanted MCSDs that rely on transcutaneous energy transmission systems and that require implanted controllers and batteries. Fully implanted assist devices such as the formally available Lion Heart (Arrow International, Inc, Reading, Pa) may diminish the incidence and consequences of sepsis and devicerelated infections, as suggested by Pae and coinvestigators ${ }^{18}$ in the multicenter Clinical Utility Baseline Study (CUBS) Trial. However, the engineering challenges and costs for fully implanted pulsatile systems remain formidable. Smaller diameter and more supple drivelines may help in the battle against infection at the percutaneous insertion site. Such modifications are feasible for rotary pump designs that do not require external venting of air. As driveline designs are optimized or eliminated and other patient management techniques advance, INTERMACS will be useful for quantifying changes in the incidence and consequences of infection.

The cumulative number of infections that occur within the first 3 months of implant and the influence of these infections on patient survival suggest that measures to diminish early infections are potentially fruitful ways for increasing survival and improving the quality of life for patients with durable MCSDs. Examples include efforts at stabilizing the driveline to optimize tissue in-growth and close follow-up of patients after hospital discharge to aggressively treat driveline infections or local non-device infections at the earliest possible time.

The referral of patients for MCSD support before the onset of critical cardiogenic shock and biventricular failure are suggested by the present analysis as ways to improve survival. This is corroborated by earlier analyses of INTERMACS data that defined markers for the onset of right 
ventricular failure as risk factors for death (eg, ascites, increased bilirubin, and increased blood urea nitrogen). Inasmuch as patients are often in cardiogenic shock when initially evaluated for MCSD support, improving the patient's condition with a period of intense medical management before VAD implant may also be of some benefit.

Surgical (intraoperative) improvements that may diminish the incidence of early postimplant infections include the use of continuous-flow (rotary) pumps that can be implanted more expeditiously than larger pulsatile pumps ${ }^{9,19,20}$ and the use of less invasive techniques, especially in patients with prior cardiac operations. ${ }^{21}$ Skull fixation for the transcutaneous power lead as reported by Westaby and associ$\operatorname{ates}^{22}$ is another possibility for decreasing percutaneous driveline infections, although this technique has not yet been used in a large number of patients.

In the rapidly evolving field of mechanical circulatory support, the INTERMACS Registry is an important tool for analyzing data that critically evaluate adverse outcomes, such as infection, and for suggesting future clinical research to expedite clinically meaningful improvements with VAD support. Clinical research to evaluate interventions that decrease the incidence of preoperative and immediate postoperative infections is critical to improving outcomes and cost for patients treated with mechanical circulatory support.

\section{References}

1. Kirklin JK, Naftel DC, Warner Stevenson L, Kormos RL, Pagani FD, Miller MA, et al. INTERMACS database for durable devices for circulatory support: first annual report. J Heart Lung Transplant. 2008;27:1065-72.

2. US Department of Health and Human Services. Artificial Heart and Assist Devices: Directions, needs, costs, societal and ethical issues. Bethesda: National Institutes of Health; 1985.

3. el-Banayosy A, Arusoglu L, Kizner L, Tenderich G, Minami K, Inoue K, et al. Novacor left ventricular assist system versus HeartMate vented electric left ventricular assist system as a long-term mechanical circulatory support device in bridging patients: a prospective study. J Thorac Cardiovasc Surg. 2000 Mar; 119:581-7.

4. Frazier OH, Myers TJ, Westaby S, Gregoric ID. Use of the Jarvik 2000 left ventricular assist system as a bridge to heart transplantation or as destination therapy for patients with chronic heart failure. Ann Surg. 2003;237:631-7.

5. Frazier OH, Rose EA, Oz MC, Dembitsky W, McCarthy P, Radovancevic B, et al. Multicenter clinical evaluation of the HeartMate vented electric left ventricular assist system in patients awaiting heart transplantation. J Thorac Cardiovasc Surg. 2001;122:1186-95.
6. Rose EA, Moskowitz AJ, Packer M, Sollano JA, Williams DL, Tierney AR, et al. The REMATCH trial: rationale, design, and end points. Ann Thorac Surg. 1999; 67:723-30.

7. Deng MC, Loebe M, el-Banayosy A, Gronda E, Jansen PG, Vigano M, et al. Mechanical circulatory support for advanced heart failure: effect of patient selection on outcome. Circulation. 2001;103:231-7.

8. Frazier OH, Rose EA, Macmanus Q, Burton NA, Lefrak EA, Poirier VL, et al. Multicenter clinical evaluation of the HeartMate 1000 IP left ventricular assist device. Ann Thorac Surg. 1992;53:1080-90.

9. Goldstein DJ. Worldwide experience with the MicroMed DeBakey Ventricular Assist Device as a bridge to transplantation. Circulation. 2003;(Suppl):108. II272-7.

10. Portner PM, Jansen PG, Oyer PE, Wheeldon DR, Ramasamy N. Improved outcomes with an implantable left ventricular assist system: a multicenter study. Ann Thorac Surg. 2001;71:205-9.

11. Lietz K, Long JW, Kfoury AG, Slaughter MS, Silver MA, Milano CA, et al. Outcomes of left ventricular assist device implantation as destination therapy in the post-REMATCH era: implications for patient selection. Circulation. 2007;116: 497-505.

12. Long JW, Kfoury AG, Slaughter MS, Silver M, Milano CA, Rogers J, et al. Longterm destination therapy with the HeartMate XVE left ventricular assist device: improved outcomes since the REMATCH study. Congest Heart Fail. 2005;11: 133-8.

13. Holman WL, Pamboukian SV, Bellot SC, Blood PS, Tallaj JA, Sharpton JL, et al. Use of an intraperitoneal ventricular assist device with a polytetrafluoroethylene barrier decreases infection. J Heart Lung Transplant. 2008;27:268-71.

14. Holman WL, Rayburn BK, McGiffin DC, Foley BA, Benza RL, Bourge RC, et al. Infection in ventricular assist devices: prevention and treatment. Ann Thorac Surg. 2003;75(suppl):S48-57.

15. Rose EA, Gelijns AC, Moskowitz AJ, Heitjan DF, Stevenson LW, Dembitsky WP, et al. Long-term use of a left ventricular assist device for endstage heart failure. N Engl J Med. 2001;345:1435-43.

16. Holman WL, Park SJ, Long JW, Weinberg A, Gupta L, Tierney AR, et al. Infection in permanent circulatory support: experience from the REMATCH Trial. $J$ Heart Lung Transplant. 2003;23:1359-65.

17. Holman WL, Kormos RL, Naftel DC, Miller MA, Pagani FD, Blume ED, et al. Predictors of death and transplant in patients with a mechanical circulatory support device: a multi-institutional study. J Heart Lung Transplant. 2009;28: 44-50.

18. Pae WE, Connell JM, Adelowo A, Boehmer JP, Korfer R, el-Banayosy A, et al. Does total implantability reduce infection with the use of a left ventricular assist device? The LionHeart experience in Europe. J Heart Lung Transplant. 2007;26: 219-29.

19. Miller LW, Pagani FD, Russell RD, John R, Boyle AJ, Aaronson KD, et al. Use of a continuous-flow device in patients awaiting heart transplantation. $N$ Engl J Med. 2007;357:885-96.

20. Schmid C, Tjan TDT, Christian E, Schmidt C, Wenzelburger F, Wilhelm M, et al. First clinical experience with the Incor left ventricular assist device. J Heart Lung Transplant. 2005;24:1188-94.

21. Frazier OH, Gregoric ID, Cohn WE. Initial experience with non-thoracic, extraperitoneal, off-pump insertion of the Jarvik 2000 heart in patients with previous median sternotomy. J Heart Lung Transplant. 2006;25:499-503.

22. Westaby S, Katsumata T, Evans R, Pigott D, Taggart DP, Jarvik RK. The Jarvik 2000 Oxford system: increasing the scope of mechanical circulatory support. J Thorac Cardiovasc Surg. 1997;114:467-74. 
Appendix E1. Durable mechanical circulatory assist devices for INTERMACS June 23, 2006, through September 30, 2008 ${ }^{\mathrm{E} 1}$

\begin{tabular}{lll}
\hline \multicolumn{1}{c}{ Company } & \multicolumn{1}{c}{ Device } & Position \\
\hline Abiomed, Inc & AbioCor TAH & TAH \\
Micromed Technology, Inc & MicroMed DeBakey VAD-Child & L \\
SynCardia Systems, Inc & SynCardia CardioWest & TAH \\
Thoratec Corporation & HeartMate II LVAS (April 2008) & L \\
& HeartMate IP & L \\
& HeartMate VE & L \\
& HeartMate XVE & L \\
& Thoratec IVAD & L/R \\
WorldHeart, Inc & NovaCor PC & L \\
& NovaCor PCq & L \\
\hline
\end{tabular}

Approved durable devices (potential for patient discharge): These devices should be entered into INTERMACS except in rare circumstances in which a patient with an approved device is in the control arm of an FDA-approval study.

\section{Appendix E2. INTERMACS Definitions for Major Infection $^{\mathrm{E} 2}$}

A clinical infection accompanied by pain, fever, drainage, and/or leukocytosis that is treated by antimicrobial agents (nonprophylactic). A positive culture from the infected site or organ should be present unless strong clinical evidence indicates the need for treatment despite negative cultures. The general categories of infection are listed below:

\section{Localized Nondevice Infection}

Infection localized to any organ system or region (eg, mediastinitis) without evidence of systemic involvement (see sepsis definition), ascertained by standard clinical methods and either associated with evidence of bacterial, viral, fungal or protozoal infection, and/or requiring empirical treatment.

\section{Percutaneous Site and/or Pocket Infection}

A positive culture from the skin and/or tissue surrounding the driveline or from the tissue surrounding the external housing of a pump implanted within the body, coupled with the need to treat with antimicrobial therapy, when there is clinical evidence of infection such as pain, fever, drainage, or leukocytosis.

\section{Internal Pump Component, Inflow, or Outflow Tract Infection}

Infection of blood-contacting surfaces of the left VAD documented by positive site culture (There should be a separate data field for paracorporeal pump that describes infection at the percutaneous cannula site, eg, Thoratec PVAD).

\section{Sepsis}

Evidence of systemic involvement by infection, manifested by positive blood cultures and/or hypotension.

\section{E-References}

E1. INTERMACS Durable Devices Included in Database. Available at: http://www uab.edu/ctsresearch/intermacs/Document $\% 20$ Library/Appendix $\% 20 \mathrm{~K} \% 20 \%$ 20Device \%20Brand \%20List, \%20FINAL, \%2010-30-08.doc 2009.

E2. INTERMACS Infection Adverse Event Definition. Available at: http://www.uab edu/ctsresearch/intermacs/Document $\% 20$ Library/Appendix $\% 20 \mathrm{~A} \% 20$-Adverse $\%$ 20Event \%20Definitions_V2.3.doc 2009. 
Infection Rates in the First Year Post MCSD Implant

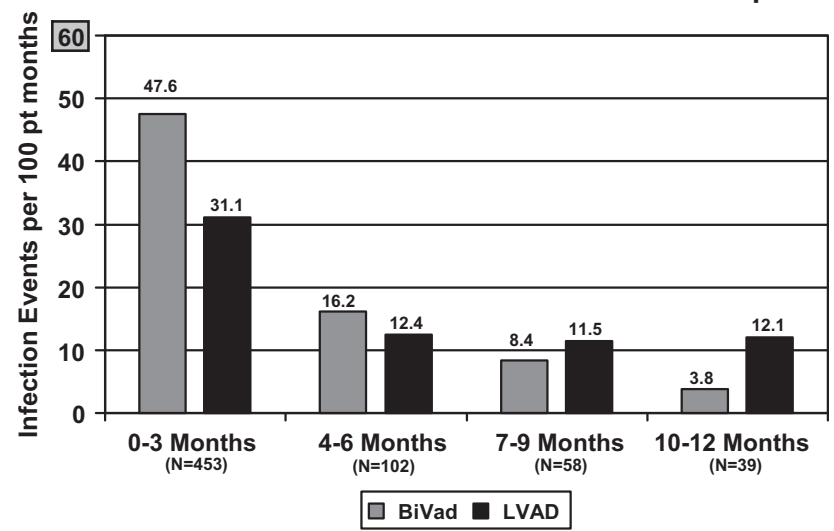

Note: Scale for infection episodes is from $0-60$ for readability purposes.

FIGURE E1. Infection rates in the first year after implantation of mechanical circulatory support device. BiVAD, Biventricular assist device; $L V A D$, left ventricular assist device.

Table E1. Sites of infection during MCSD support (INTERMACS June 23, 2006-September 30, 2008): Primary adult pulsatile implants

\begin{tabular}{lccc}
\hline \multicolumn{3}{c}{ Infections } \\
\hline Device side & Patients & Events (patients) & Prevalence (\%) \\
\hline LVAD & 465 & $563(190)$ & 41 \\
BiVAD & 128 & $119(54)$ & 42 \\
Total & 593 & $682(244)$ & \\
\hline
\end{tabular}

$L V A D$, Left ventricular assist device; $B i V A D$, biventricular assist device.

Table E2. Gender, race, and age at implant (INTERMACS June 23, 2006-September 30, 2008): Primary adult pulsatile implants

\begin{tabular}{lc}
\hline Gender & \\
Male & $479(80.8)$ \\
Female & $114(19.2 \%)$ \\
Race & \\
White & $425(71.7 \%)$ \\
African American & $126(21.2 \%)$ \\
Other & $42(7.1 \%)$ \\
Age at implant (y) & \\
Mean & 51.5 \\
Range & $19.8-79.0$ \\
\hline
\end{tabular}

Table E3. Sites of infection on pulsatile MCSD support (INTERMACS June 23, 2006-September 30, 2008): Primary adult pulsatile implants

\begin{tabular}{lrc}
\hline \multicolumn{1}{c}{ Location* } & n & \% of $\mathbf{6 8 2}$ \\
\hline Pump-related & & \\
$\quad$ Driveline & 140 & 21 \\
Pump pocket & 51 & 7 \\
$\quad$ Pump interior & 3 & 0.4 \\
Line sepsis & 46 & 7 \\
Mediastinum & 18 & 3 \\
Peripheral wound & 22 & 3 \\
Pulmonary & 116 & 17 \\
Gastrointestinal & 39 & 6 \\
Urinary tract & 111 & 16 \\
Positive blood cultures & 218 & 32 \\
Other & 100 & 15 \\
Unknown & 7 & 1 \\
Total & 682 & \\
\hline
\end{tabular}

*More than one location can exist for one identified infection.
Table E4. Preimplant risk factors for cumulative infections (INTERMACS June 23, 2006-September 30, 2008): Primary adult pulsatile implants)

\begin{tabular}{lcc}
\hline \multicolumn{1}{c}{ Risk factor } & Hazard ratio & $\boldsymbol{P}$ value \\
\hline BiVAD & 1.35 & .04 \\
Age (older) & $1.25^{*}$ & $<.0001$ \\
INTERMAC level 1 & 2.34 & $<.0001$ \\
Blood urea nitrogen (higher) & $2.26 \dagger$ & $<.0001$ \\
\hline
\end{tabular}

$B i V A D$, Biventricular assist device. ${ }^{*}$ The hazard ratio is calculated for a 10 -year increase in age. $\dagger$ The hazard ratio is calculated for a 50-unit increase in blood urea nitrogen. 